\title{
UNIFORMLY ERGODIC MULTIOPERATORS
}

\author{
M. MBEKHTA AND F.-H. VASILESCU
}

ABSTRACT. A version of the uniform ergodic theorem valid for commuting multioperators is given.

Let $X$ be a complex Banach space, and let $\mathscr{L}(X)$ be the algebra of linear continuous operators acting in $X$. For every $S \in \mathscr{L}(X)$ we set

$$
M_{k}(S)=k^{-1}\left(I+S+S^{2}+\cdots+S^{k-1}\right), \quad k=1,2,3, \ldots,
$$

i.e. the averages associated with $S$, where $I=I_{X}$ is the identity of $X$.

If $T=\left(T_{1}, \ldots, T_{n}\right) \in \mathscr{L}(X)^{n}$ is a commuting multioperator (briefly, a c.m.) we also set

$$
M_{\nu}(T)=M_{\nu_{1}}\left(T_{1}\right) \cdots M_{\nu_{n}}\left(T_{n}\right), \quad \nu \in \mathbf{Z}_{+}^{n}, \nu \geq e,
$$

where $\mathbf{Z}_{+}^{n}$ is the family of multi-indices of length $n$ (i.e. $n$-tuples of nonnegative integers) and $e:=(1, \ldots, 1) \in \mathbf{Z}_{+}^{n}$. In other words, (2) defines the averages associated with $T$ (see [Bru], [Du-Sc], [Kr], etc.).

Definition. A c.m. $T \in \mathscr{L}(X)^{n}$ is said to be Cesàro quasi-bounded if the sequences

$$
\left(\prod_{k \neq j} M_{\nu_{k}}\left(T_{k}\right)\right)_{\nu_{1} \geq 1, \ldots, \nu_{j-1} \geq 1, \nu_{j+1} \geq 1, \ldots, \nu_{n} \geq 1}(j=1, \ldots, n)
$$

are bounded in $\mathscr{L}(X)$. If, in addition, the limit

$$
\lim _{\nu} M_{\nu}(T)
$$

exists in the uniform topology of $\mathscr{L}(X)$, then $T$ is said to be uniformly ergodic.

Remarks. (1) If $n=1$, then (3) is automatically fulfilled, and therefore the above definition extends the usual concept of uniformly ergodic operator (see, for instance, $[\mathrm{Kre}])$.

(2) A pair $T=\left(T_{1}, T_{2}\right)$ is Cesàro quasi-bounded if and only if both $T_{1}$ and $T_{2}$ are Cesàro bounded, i.e. $\left(M_{k}\left(T_{j}\right)\right)_{k \geq 1}(j=1,2)$ are bounded [Kre].

(3) If $T_{1}, \ldots, T_{n}$ are uniformly ergodic commuting operators, then $T=$ $\left(T_{1}, \ldots, T_{n}\right)$ is uniformly ergodic. The converse is not true, in general. Indeed,

Received by the editors January 24, 1994 and, in revised form, March 24, 1994; originally communicated to the Proceedings of the AMS by Palle E. T. Jorgensen.

1991 Mathematics Subject Classification. Primary 47A35; Secondary 47A13.

Key words and phrases. average, Cesàro quasi-boundedness. 
if $T_{1}=0$ and $T_{2}$ is Cesàro bounded but not uniformly ergodic (see [Mb-Ze] for such an example), then $T=\left(T_{1}, T_{2}\right)$ is, however, uniformly ergodic.

(4) A c.m. $T \in \mathscr{L}(X)^{n}$ is Cesàro quasi-bounded if and only if $T^{*} \in \mathscr{L}\left(X^{*}\right)^{n}$ is Cesàro quasi-bounded, where $X^{*}$ is the dual of $X$ and $T^{*}$ is the "adjoint" of $T$ (i.e. $\left.T^{*}=\left(T_{1}^{*}, \ldots, T_{n}^{*}\right)\right)$. Similarly, $T$ is uniformly ergodic if and only if $T^{*}$ is uniformly ergodic.

(5) It is not known to the authors whether the existence of the limit (4) implies the Cesàro quasi-boundedness if $n \geq 2$. Note that the current hypothesis when defining the averages (2) is that $T_{1}, \ldots, T_{n}$ is a family of commuting power bounded operators (see [Kre]).

Let $T \in \mathscr{L}(X)^{n}$ be a c.m. We define the operators $R_{\nu, j}(T) \in \mathscr{L}(X)$ by the formula

$$
R_{\nu, j}(T):=\nu_{j}^{-1} T_{j}^{\nu_{j}} \prod_{k \neq j} M_{\nu_{k}}\left(T_{k}\right), \quad \nu \geq e, j=1, \ldots, n, n \geq 2 .
$$

If $n=1$, we set $R_{\nu, 1}(T):=\nu_{1}^{-1} T_{1}^{\nu_{1}}$.

Lemma 1. If $T \in \mathscr{L}(X)^{n}$ is Cesàro quasi-bounded, then $\lim _{\nu} M_{\nu}(T)\left(I-T_{j}\right)=0$ if and only if $\lim _{\nu} R_{\nu, j}(T)=0$ for each $j \in\{1, \ldots, n\}$.

Proof. As we have

$$
M_{\nu_{j}}\left(T_{j}\right)\left(I-T_{j}\right)=\nu_{j}^{-1}\left(I-T_{j}^{\nu_{j}}\right),
$$

it follows that

$$
\begin{aligned}
M_{\nu}(T)\left(I-T_{j}\right) & =\nu_{j}^{-1}\left(I-T_{j}^{\nu_{j}}\right) \prod_{k \neq j} M_{\nu_{k}}\left(T_{k}\right) \\
& =\nu_{j}^{-1} \prod_{k \neq j} M_{\nu_{k}}\left(T_{k}\right)-R_{\nu, j}(T) .
\end{aligned}
$$

Since $T$ is assumed to be Cesàro quasi-bounded, we have

$$
\lim _{\nu} \nu_{j}^{-1} \prod_{k \neq j} M_{\nu_{k}}\left(T_{k}\right)=0,
$$

which combined with (6) leads easily to our assertion.

Remark. If $T \in \mathscr{L}(X)^{n}$ is Cesàro quasi-bounded, and if $\lim _{k} k^{-1} T_{j}^{k}=0$ for some $j$, then $\lim _{\nu} R_{\nu, j}(T)=0$.

For every operator $S \in \mathscr{L}(X)$ we denote by $N(S)$ the kernel of $S$.

The next result is a version of [Mb-Ze, Lemma 1].

Lemma 2. Let $T \in \mathscr{L}(X)^{n}$ be Cesàro quasi-bounded, such that $\lim _{\nu} R_{\nu, j}(T)=$ $0(j=1, \ldots, n)$. Then

$$
\left[\sum_{j=1}^{n}\left(I-T_{j}\right) X\right] \cap\left[\bigcap_{j=1}^{n} N\left(I-T_{j}\right)\right]=\{0\} .
$$

Proof. Let $x$ be a vector in the left-hand side of (7). Then $x=\sum_{j=1}^{n}\left(I-T_{j}\right) x_{j}$, and so

$$
M_{\nu}(T) x=\sum_{j=1}^{n} M_{\nu}(T)\left(I-T_{j}\right) x_{j}
$$


But we also have $x=T_{j} x(j=1, \ldots, n)$, whence $M_{\nu}(T) x=x$. Therefore

$$
\begin{aligned}
x & =\lim _{\nu} M_{\nu}(T) x=\lim _{\nu} \sum_{j=1}^{n} M_{\nu}(T)\left(I-T_{j}\right) x_{j} \\
& =\lim _{\nu} \sum_{j=1}^{n} R_{\nu, j}(T) x_{j}=0,
\end{aligned}
$$

via Lemma 1.

The standard decomposition of the space with respect to a uniformly ergodic c.m. can be expressed as follows.

Lemma 3. Let $T \in \mathscr{L}(X)^{n}$ be uniformly ergodic, and let $E:=\lim _{\nu} M_{\nu}(T)$. Then $E$ is a projection in $X$ such that

$$
\begin{gathered}
E X=\bigcap_{j=1}^{n} N\left(I-T_{j}\right), \\
N(E)=\left[\sum_{j=1}^{n}\left(I-T_{j}\right) X\right]^{-} .
\end{gathered}
$$

Proof. Note the identity

$$
T_{j} M_{\nu}(T)=\frac{\nu_{j}+1}{\nu_{j}} M_{\nu+e_{j}}(T)-\frac{1}{\nu_{j}} \prod_{k \neq j} M_{\nu_{k}}\left(T_{k}\right),
$$

valid for every $j=1, \ldots, n$, where

$$
e_{j}=(\underbrace{0, \ldots, 0,1}_{j}, 0, \ldots, 0) \text {. }
$$

Passing to the limit in (10), we obtain

$$
T_{j} E=E T_{j}=E, \quad j=1, \ldots, n .
$$

As we have from (11) that $E M_{\nu}(T)=E$, it follows that $E^{2}=E$, i.e. $E$ is a projection.

To prove (8), let $x=E y$. Then, from (11),

$$
\left(I-T_{j}\right) x=E\left(I-T_{j}\right) y=0, \quad j=1, \ldots, n,
$$

thus $E X \subset \bigcap_{j=1}^{n} N\left(I-T_{j}\right)$.

Conversely, if $\left(I-T_{j}\right) x=0$ for all $j$, then $x=M_{\nu}(T) x$ for all $\nu$, and so $x=E X$. Consequently $\bigcap_{j=1}^{n} N\left(I-T_{j}\right) \subset E X$, showing that (8) holds.

Let us prove (9). The inclusion $\sum_{j=1}^{n}\left(I-T_{j}\right) X \subset N(E)$ follows easily from (11). Conversely, if

$$
x^{*} \in\left[\sum_{j=1}^{n}\left(I-T_{j}\right) X\right]^{\perp},
$$

then $x^{*}\left(I-T_{j}\right)=0$ for each $j$. Thus $x^{*} M_{\nu}(T)=x^{*}$ for all $\nu$, whence $x^{*}=x^{*} E$. Consequently $x^{*} \in N(E)^{\perp}$, showing that

$$
N(E) \subset\left[\sum_{j=1}^{n}\left(I-T_{j}\right) X\right]^{-},
$$

which completes the proof of the lemma. 
The next result extends to several variables an important observation of [Lin]. Lemma 4. Let $T \in \mathscr{L}(X)^{n}$ be uniformly ergodic. Then the space $\sum_{j=1}^{n}\left(I-T_{j}\right) X$ is closed in $X$.

Proof. Let $Y:=\left[\sum_{j=1}^{n}\left(I-T_{j}\right) X\right]^{-}$. By the previous lemma, $Y=(I-E) X$. Note that $Y$ is invariant under $T$. Let $S=T \mid Y$. Then

$$
M_{\nu}(S)=M_{\nu}(T)(I-E) \mid Y,
$$

and therefore

$$
\left\|M_{\nu}(S)\right\| \leq\left\|M_{\nu}(T)(I-E)\right\| .
$$

Since $\lim _{\nu} M_{\nu}(T)(I-E)=0$, we can find a multi-index $\nu$ such that $\left\|M_{\nu}(S)\right\|<$ 1. Then $I-M_{\nu}(S)$ is invertible. We deduce from here that $e \notin \sigma(T)$, where $\sigma(S)$ is the (Taylor) joint spectrum of $S$ (see [Tay 1], or [Vas]). Indeed if $e \in \sigma(S)$, as we have

$$
\sigma\left(I-M_{\nu}(S)\right)=\left\{1-M_{\nu}(z) ; z \in \sigma(S)\right\}
$$

by the spectral mapping theorem (see [Tay 2] or [Vas]), we obtain $0 \in$ $\sigma\left(I-M_{\nu}(S)\right)$, which is a contradiction. But if we have $e \notin \sigma(S)$, then, in particular, $\sum_{j=1}^{n}\left(I-S_{j}\right) Y=Y$, which follows from the definition of the (Taylor) joint spectrum. Therefore

$$
Y=\sum_{j=1}^{n}\left(I-S_{j}\right) Y \subset \sum_{j=1}^{n}\left(I-T_{j}\right) X \subset Y,
$$

which establishes our assertion.

The next result is a several variables version of the uniform ergodic theorem from [Dun], which reflects the improvement due to [Lin].

Theorem 1. Let $T \in \mathscr{L}(X)^{n}$ be Cesàro quasi-bounded. Then the following two conditions are equivalent.

(i) $T$ is uniformly ergodic.

(ii) $\lim _{\nu} R_{\nu, j}(T)=0(j=1, \ldots, n)$, and the subspaces $\sum_{j=1}^{n}\left(I-T_{j}\right) X$, $\sum_{j=1}^{n}\left(I^{*}-T_{j}^{*}\right) X^{*}$ are closed in $X, X^{*}$, respectively.

Proof (i) $\Rightarrow$ (ii). If $T$ is uniformly ergodic, then $\lim _{\nu} M_{\nu}(T)\left(I-T_{j}\right)=0$, and so $\lim _{\nu} R_{\nu, j}(T)=0(j=1, \ldots, n)$, by Lemma 1 . The space $\sum_{j=1}^{n}\left(I-T_{j}\right) X$ is closed by Lemma 4.

Since $T^{*}$ is also uniformly ergodic, the space $\sum_{j=1}^{n}\left(I^{*}-T_{j}^{*}\right) X^{*}$ is closed too.

(ii) $\Rightarrow$ (i). Let $X^{n}=X \oplus \cdots \oplus X \quad(n$ times), endowed with the norm $\left\|x_{1} \oplus \cdots \oplus x_{n}\right\|^{2}=\left\|x_{1}\right\|^{2}+\cdots+\left\|x_{n}\right\|^{2}$.

Let $\delta_{T}: X^{n} \rightarrow X$ be given by the formula

$$
\delta_{T}\left(\bigoplus_{j=1}^{n} x_{j}\right):=\sum_{j=1}^{n}\left(I-T_{j}\right) x_{j}, \quad x_{1}, \ldots, x_{n} \in X .
$$

Let also $\gamma_{T}: X \rightarrow X^{n}$ be given by

$$
\gamma_{T}(x):=\bigoplus_{j=1}^{n}\left(I-T_{j}\right) x, \quad x \in X .
$$


If $\delta_{T^{*}}, \gamma_{T^{*}}$ are the operators given by (12), (13) for $T^{*}$, respectively, then we have

$$
\delta_{T^{*}}=\left(\gamma_{T}\right)^{*}, \quad \gamma_{T^{*}}=\left(\delta_{T}\right)^{*}
$$

as one can easily check (via the natural identification $X^{* n}=X^{n *}$, described in [Kat]).

The hypothesis implies that the spaces $Y:=\delta_{T}\left(X^{n}\right), Y_{*}:=\delta_{T^{*}}\left(X^{* n}\right)$ are closed. If $Z:=N\left(\gamma_{T}\right), Z_{*}=N\left(\gamma_{T^{*}}\right)$, then $Y_{*}=Z^{\perp}$ and $Z_{*}=Y^{\perp}$, by (14) and the fact that $Y, Y_{*}$ are closed.

By virtue of Lemma 2, we have $Y_{*} \cap Z_{*}=\{0\}$. Therefore $(Y+Z)^{-}=X$. Then for each $x \in X$ we find sequences $\left(y_{k}\right)_{k} \subset Y,\left(z_{k}\right)_{k} \subset Z$ such that $y_{k}+z_{k} \rightarrow x$ as $k \rightarrow \infty$. Then

$$
\gamma_{T}(x)=\lim _{k \rightarrow \infty} \gamma_{T}\left(y_{k}+z_{k}\right)=\lim _{k \rightarrow \infty} \gamma_{T}\left(y_{k}\right),
$$

and therefore

$$
\gamma_{T}(x) \in \overline{\gamma_{T}(Y)}, \quad x \in X .
$$

We shall show that $\gamma_{T}(Y)$ is closed in $X^{n}$. Indeed, let $S=T \mid Y$. Since $Y=\delta_{T}\left(X^{n}\right)$ is closed, there is a constant $C>0$ such that for every $y \in Y$ we can find $x_{1}, \ldots, x_{n} \in X$ such that $y=\delta_{T}\left(x_{1} \oplus \cdots \oplus x_{n}\right)$ and $\left\|x_{1} \oplus \cdots \oplus x_{n}\right\| \leq$ $C\|y\|$.

Hence

$$
\begin{aligned}
\left\|M_{\nu}(S) y\right\| & =\left\|\sum_{j=1}^{n} M_{\nu}(T)\left(I-T_{j}\right) x_{j}\right\| \\
& \leq C\left(\sum_{j=1}^{n}\left\|M_{\nu}(T)\left(I-T_{j}\right)\right\|^{2}\right)^{1 / 2}\|y\|
\end{aligned}
$$

As we have

$$
\lim _{\nu}\left(\sum_{j=1}^{n}\left\|M_{\nu}(T)\left(I-T_{j}\right)\right\|^{2}\right)^{1 / 2}=\lim _{\nu}\left(\sum_{j=1}^{n}\left\|R_{\nu, j}(T)\right\|^{2}\right)^{1 / 2}=0
$$

by Lemma 1, it follows from (16) that we can find a multi-index $\nu$ such that $\left\|M_{\nu}(S)\right\|<1$.

Now, an argument used in the proof of Lemma 4 shows that $e \notin \sigma(S)$. In particular, as a consequence of the definition of the (Taylor) joint spectrum, $\gamma_{S}$ has closed range, and so $\gamma_{T}(Y)$ is closed.

From (15) we can now derive that for every $x \in X$ there is a $y \in Y$ such that $\gamma_{T}(x)=\gamma_{T}(y)$. Hence $z:=x-y \in Z$, and so $X=Y+Z$. As we have $Y \cap Z=\{0\}$ by Lemma 2, we have obtained that $X$ is the direct topological sum of the spaces $Y, Z$.

We have only to show that $\lim _{\nu} M_{\nu}(T)$ exists in $\mathscr{L}(X)$. Let $E$ be the projection of $X$ onto $Z$, parallel to $Y$. Since $M_{\nu}(T \mid Z)=I_{Z}$, we have for all $\nu^{\prime}, \nu^{\prime \prime} \in \mathbf{Z}_{+}^{n}$ :

$$
\begin{gathered}
\left\|\left[M_{\nu^{\prime}}(T)-M_{\nu^{\prime \prime}}(T)\right] x\right\|=\left\|\left[M_{\nu^{\prime}}(S)-M_{\nu^{\prime \prime}}(S)\right](I-E) x\right\| \\
\leq(1+\|E\|)\left\|M_{\nu^{\prime}}(S)-M_{\nu^{\prime \prime}}(S)\right\|\|x\| .
\end{gathered}
$$


As $\lim _{\nu} M_{\nu}(S)=0$ by (16) and (17), we obtain from (18) that the limit $\lim _{\nu} M_{\nu}(T)$ exists in $\mathscr{L}(X)$. Therefore $T$ is uniformly ergodic and the proof of the theorem is completed.

Remark. The space $\sum_{j=1}^{n}\left(I^{*}-T_{j}^{*}\right) X^{*}$ is closed if and only if the space

$$
\left\{\bigoplus_{j=1}^{n}\left(I-T_{j}\right) x \in X^{n} ; x \in X\right\}
$$

is closed. This follows from the fact that (19) is precisely $\gamma_{T}(X)$. This shows that for $n=1$, condition (ii) from Theorem 1 is equivalent to $\lim _{k} k^{-1} T_{1}^{k}=0$ and $\left(I-T_{1}\right) X$ closed, as in Lin's version of the uniform ergodic theorem (see [Lin]).

If $n>1$, then the closedness of the space $\sum_{j=1}^{n}\left(I-T_{j}\right) X$ and that of (19) are independent. We owe to V. Müller (Prague) an example of this, which will be published elsewhere.

For various purposes, one can state another (less concentrated) version of Theorem 1, also reflecting more recent contributions for the case of one operator (see, for instances, [Mb-Ze]). Unfortunately, conditions expressed in terms of poles or finite descent do not seem to have a counterpart in our context.

Theorem 2. Let $T \in \mathscr{L}(X)^{n}$ be Cesàro quasi-bounded, such that $\lim _{\nu} R_{\nu, j}(T)=$ $0(j=1, \ldots, n)$. Then the following conditions are equivalent:

(a) $T$ is uniformly ergodic.

(b) The spaces

$$
\begin{gathered}
\sum_{j=1}^{n}\left(I-T_{j}\right) X+\bigcap_{j=1}^{n} N\left(I-T_{j}\right), \\
\sum_{j=1}^{n}\left(I^{*}-T_{j}^{*}\right) X^{*}+\bigcap_{j=1}^{n} N\left(I^{*}-T_{j}^{*}\right)
\end{gathered}
$$

are closed in $X, X^{*}$, respectively.

(c) The spaces $\sum_{j=1}^{n}\left(I-T_{j}\right) X, \sum_{j=1}^{n}\left(I^{*}-T_{j}^{*}\right) X^{*}$ are closed in $X, X^{*}$, respectively.

(d) The space $\sum_{j=1}^{n}\left(I-T_{j}\right) X$ is closed and complemented in $X$, and its complement is equal to $\bigcap_{j=1}^{n} N\left(I-T_{j}\right)$.

(e) There are two closed subspaces $Y, Z$ of $X$ that are invariant under $T$, such that $X=Y+Z, \lim _{\nu} M_{\nu}(T \mid Y)=0$ and $T \mid Z=\left(I_{Z}, \ldots, I_{Z}\right)$.

Proof. (a) $\Rightarrow$ (b). It follows from (8), (9) and Lemma 4 that

$$
X=\sum_{j=1}^{n}\left(I-T_{j}\right) X+\bigcap_{j=1}^{n} N\left(I-T_{j}\right) .
$$

In particular, the right-hand side of $(20)$ is closed.

The remaining assertion from (b) follows similarly, by the fact that $T^{*}$ is also uniformly ergodic.

(b) $\Rightarrow$ (c) Let $X_{0}$ be the right-hand side of (20), which is closed by the 
hypothesis. If $\delta_{T}$ is the operator (12), then the mapping

given by

$$
\tau:\left[X^{n} / N\left(\delta_{T}\right)\right] \oplus\left[\bigcap_{j=1}^{n} N\left(I-T_{j}\right)\right] \rightarrow X_{0}
$$

$$
\tau\left(\left[\bigoplus_{j=1}^{n} x_{j}+N\left(\delta_{T}\right)\right] \oplus z\right)=\sum_{j=1}^{n}\left(I-T_{j}\right) x_{j}+z
$$

is linear, continuous and bijective, via Lemma 2.

Therefore $\tau^{-1}$ is continuous, and $\sum_{j=1}^{n}\left(I-T_{j}\right) X$ must be closed in $X$.

A similar argument shows that $\sum_{j=1}^{n}\left(I^{*}-T_{j}^{*}\right) X^{*}$ must be closed in $X^{*}$.

(c) $\Rightarrow$ (d). This follows from the proof of the implication (ii) $\Rightarrow$ (i) of Theorem 1 .

(d) $\Rightarrow\left(\right.$ e). We take $Y:=\sum_{j=1}^{n}\left(I-T_{j}\right) Y$ and $Z:=\bigcap_{j=1}^{n} N\left(I-T_{j}\right)$. Then $T \mid Z=\left(I_{Z}, \ldots, I_{Z}\right)$, and $X=Y+Z$, as in the proof Theorem 1. That $\lim _{\nu} M_{\nu}(T \mid Y)=0$ follows from (16) and (17).

(e) $\Rightarrow(a)$. Since $\lim _{\nu} M_{\nu}(T \mid Y)=0$, it follows as in the proof of Theorem 1 that $e \neq \sigma(T \mid Y)$.

On the other hand, $\sigma(T \mid Z)=\{e\}$. It follows from the Taylor idempotent theorem (see [Tay 2] or [Vas]) that $Y \cap Z=\{0\}$. The existence of the limit $\lim _{\nu} M_{\nu}(T)$ can be obtained as in the last part of the proof of Theorem 1.

Hence all equivalences are established.

A version of [Lin, Corollary 1] can be also obtained in our context.

Corollary. Let $T \in \mathscr{L}(X)^{n}$ be Cesàro quasi-bounded, such that $\lim _{\nu} R_{\nu, j}(T)=$ $0(j=1, \ldots, n)$. Suppose that

$$
\begin{aligned}
\max _{1 \leq j \leq n} \sup _{m \geq 0}\left\|\sum_{k=0}^{m} T_{j}^{k} y\right\|<\infty, & y \in\left[\sum_{j=1}^{n}\left(I-T_{j}\right) X\right]^{-}, \\
\max _{1 \leq j \leq n} \sup _{m \geq 0}\left\|\sum_{k=0}^{m} T_{j}^{* k} y^{*}\right\|<\infty, & \left.y^{*} \in\left[\sum_{j=1}^{n}\left(I^{*}-T_{j}^{*}\right) X\right)^{*}\right]^{-} .
\end{aligned}
$$

Then $T$ is uniformly ergodic.

Proof. By the uniform boundedness principle, we have

$$
\max _{1 \leq j \leq n} \sup _{m \geq 0}\left\|\sum_{k=0}^{m} S_{j}^{k}\right\|<\infty,
$$

where $S_{j}:=T_{j} \mid Y(j=1, \ldots, n), Y:=\left[\sum_{j=1}^{n}\left(I-T_{j}\right) X\right]^{-}$. Then

$$
\lim _{k \rightarrow \infty} M_{k}\left(S_{j}\right)=0 \quad(j=1, \ldots, n),
$$

and therefore $\lim _{\nu} M_{\nu}(S)=0$.

In particular, we obtain $\bigcap_{j=1}^{n} N\left(I_{Y}-S_{j}\right)=\{0\}$. It follows from Theorem 2 (see (d) and (e)) that

$$
\sum_{j=1}^{n}\left(I-S_{j}\right) Y=Y \subset \sum_{j=1}^{n}\left(I-T_{j}\right) X \subset Y .
$$


Therefore $\sum_{j=1}^{n}\left(I-T_{j}\right) X$ is closed. Similarly, $\sum_{j=1}^{n}\left(I^{*}-T_{j}^{*}\right) X^{*}$ is closed. In virtue of Theorem 1, $T$ is uniformly ergodic.

\section{ACKNOWLEDGMENT}

This work was carried out while the second-named author was visiting the University of Lille 1, France. He would like to express his gratitude for the hospitality of that institution.

\section{REFERENCES}

[Bru] A. Brunel, Théorème ergodique ponctuel pour un semi-groupe commutatif finiment engendré de contractions de $L^{1}$, Ann. Inst. Henri Poincare 9 (1973), 327-343.

[Dun] N. Dunford, Spectral theory, I: Convergence to projections, Trans. Amer. Math. Soc. 54 (1943), 185-217.

[Du-Sc] N. Dunford and J. T. Schwartz, Linear operators, I: General theory, Interscience, New York, 1958.

[Kat] T. Kato, Perturbation theory for linear operators, Springer-Verlag, Berlin, Heidelberg and New York, 1976.

[Kre] U. Krengel, Ergodic theorems, De Gruyter, Berlin and New York, 1985.

[Lin] M. Lin, On the uniform ergodic theorem, Proc. Amer. Math. Soc. 43 (1974), 337-340.

[Mb-Ze] M. Mbekhta and J. Zemànek, Sur le théorème ergodique uniforme et le spectre, $\mathrm{C}$. R. Acad. Sci. Paris Sér. I 317 (1993), 1155-1158.

[Tay 1] J. L. Taylor, A joint spectrum for several commuting operators, J. Funct. Anal. 6 (1970), 172-191.

[Tay 2] _ , The analytic functional calculus for several commuting operartors, Acta Math. 125 (1970), 1-38.

[Vas] F.-H. Vasilescu, Analytic functional calculus and spectral decompositions, Editura Academiei, Bucuresti, and Reidel, Dordrecht, 1982.

U.F.R. De Mathématiques, Université des Sciences et Technologies de Lille, 59655Villeneuve d'Asce Cedex, France

E-mail address: mbekhta@gat.univ-lille1.fr

INSitute of Mathematics of the Romanian ACademy, P.O. Box 1-764, 70700 Bucharest, ROMANIA

Current address: U.F.R. de Mathématiques, Université des Sciences et Technologies de Lille, 59655 Villeneuve D'Ascq cedex, France

E-mail address: fhvasilegat.univ-lille1.fr 